\title{
Discussion to 55 Clinical Application of Ophthalmophonogram in Carotid-Cavernous Fistula
}

\author{
Hiroyuki Tanji, Kazuo Watanabe, Katsuhiko Yamaguchi \\ Hiroshi Suda, Tsuneharu Higa, Sinichiro Endow \\ 2nd Department of Surgery, Fukushima Medical College
}

There has been outlined a comprehensive plan of surgical attack on the carotid cavernous fistula, namely (1) intracranial carotid and ophthalmic artery clipping, immediately followed by (2) muscle emolization of the internal carotid artery, and (3) ligation and section of the common, internal and external carotid arteries, But there are many problems in these procedures.

We recorded bruit as phonogram over the eye, named it Ophthalmophonogram (O. P. G.) and studied its clinical applications. Bruit is the figure of motor energy generated by turburance produced by hypoplasticity of laminal flow caused by abnormal hemodynamics, so bruit provides a barometer of activity of fistula.

Case I. a 64-year-old woman developed spontaneous pulsating exophthalmos. The bruit was heard over the right eye, synchronously with the heart beat. Case 2 . a 28-year-old woman had sustained head injury 4 months ago, and developed left exophthalmos and headache. The bruit was heard over the left eye by stethoscope. Case 3. a 31-year-old man developed loss of vision followed by head injruy and complained of continuous tinnitis. Carotid angiogram showed right carotid cavernous fistula.

The pathways of drainge of the fistula of three patients were same, and carotid angiogram showed arterial blood supply from the carotid artery into the cavernous sinus and the superior ophthalmic vein, and cross circulation from opposite carotid artery was noticed.

We recorded both bruit and PCG through filter L, M, H, E which had each chracteristic frequency, by means of fixed microphone of phonoelectrocardioscope over the eye of three patients and normal adults, in a supine position, in closing both eyes and during temporary stopping respiratory movement.

There were no pathological murmurs in O. P. G. of the normal adults, but characteristic murmurs were recorded in O. P. G. of three patients, namely, high pitched, systolic and diastolic, machinary murmurs. In case 1 . high amplitude machinary murmurs in right O. P. G. which had been noticed preoperatively, disappeared following cervical ligation of right common, internal and external carotid artery. In case 2 . low amplitude machinary murmurs were recorded on left side and in case 3. high amplitude machinary muramurs were recorded on both sides, especially on right side through filter M. (150 cps) and H. (300 cps.)

Although analysis of murmurs in O. P. G. is difficult problems, we think that if O. P. G. is recorded by smaller microphone which has higher sensitivity, the findings of $\mathrm{O}$. P. G. should be more useful as index of objective data during preoperative and postoperative procedures. 